\title{
GENERATION OF DIAGNOSTIC X-RAY RADIATION QUALITIES IN THE SSDL X-RAY SYSTEM ACCORDING TO IEC 61267 STANDARD
}

\author{
Doğan Yaşara $^{a^{*}}$, Enis Kapdana, Hasan Erez ${ }^{a}$, Tülin Zengin ${ }^{a}$, Erol Kam ${ }^{b}$ \\ ${ }^{a}$ TAEK,-Çekmece Nuclear Research Center, Küçükçekmece, İstanbul, Turkey \\ dogan.yasar@taek.gov.tr*(corresponding author) \\ ${ }^{b}$ Faculty of Arts \& Science, Ylldiz Technical University, İstanbul, Turkey
}

\begin{abstract}
Ionizing radiation metrology laboratories generate their own gamma and $\mathrm{x}$-ray radiation qualities with respect to reference radiations generated by international standards, such as IAEA (International Atomic Energy Agency) used for the radiotherapy field, ISO (International Organization for Standardization) used for the radiation protection field and IEC (International Electrotechnical Commission) used for the diagnostic field. IEC constructed standards of four reference radiation qualities (RQR, RQA, RQC and RQT) representing radiation energy qualities used in the diagnostic radiology. In this regard, SSDL (Secondary Standard Dosimetry Laboratories), in parallel with developments in the diagnostic radiology, have desire to carry out the calibration and test responsibility of dosimetry and measurement devices to be used in the diagnostic radiology by generating their own radiation energy qualities and reference dosimetry standards.
\end{abstract}

The first step of this study is to obtain $\mathrm{x}$-ray radiation qualities. Diagnostic RQR radiation qualities represent radiation beams applied to patients in general radiography, fluoroscopy and dental practices. RQR radiation qualities were obtained according to criteria specified in IEC standard.

According to IEC standard, half value layer (HVL) filtered and unfiltered air kerma dose rates are predicted to be a value between 0.485 and 0.515 . In our study, those values were found to be between 0.497 and 0.506 . Reliability and conformity of obtained qualities were determined by using various measurement techniques.

In the second step of the study, after obtaining reference standard dosimetry calibrated in PSDL, which will constitute the national standard, the international standardization will be extended throughout the country 
by providing calibration services for dosimetry and measurement devices to the users in the diagnostic radiology.

Keywords: X-ray, radiation quality, dosimetry, diagnostic radiology, calibration

\section{Introduction}

Among artificial sources, medical ionizing radiation sources make the highest contribution to population dose and approximately $90 \%$ of such contribution consists of diagnostic x-rays. One of the reasons for this is that the number of $x$-ray examinations increases every year [1]. During development of technology, many types of ionizing radiation are increasingly becoming a part of society day by day. Medical imaging techniques are essential methods used to diagnose diseases and injuries. During the use of x-ray imaging systems, radiation doses must be kept under control by designing $\mathrm{x}$-ray imaging systems ideally.

The International Commission on Radiological Protection (ICRP) makes suggestions for the justification and optimization of all medical practices with respect to principals of radiation safety, as well as the use of dose limitations in practices using current diagnostic methods [2]. The International Electrotechnical Commission (IEC) is an international organization preparing and publishing standards to solve possible problems in electrical and electrical-related technologies. This international organization published the IEC 61267 standard, which reveals special conditions for the characteristics of x-ray radiation used in tests and calibrations of devices [3].

Various diagnostic techniques are applied in $\mathrm{x}$-ray diagnostic radiology: fluoroscopy, interventional radiologic methods, mammography, computerized tomography (CT), dental and general radiography. X-ray beam having a tube voltage between 20 and $150 \mathrm{kV}$ is used in such methods. For instance, fluoroscopy, CT, dental and general radiography practices use tube voltages between 40 and $150 \mathrm{kV}$ and tungsten the anode material. Mammographic investigations are conducted in tube voltages between 22 and $40 \mathrm{kV}$ and they usually use molybdenum anode and filter. The purpose of dosimetry in diagnostic and interventional radiology - as a part of quality safety process - is to evaluate the hardware performance. Some laboratories use various inappropriate radiation qualities and standards $[3,4,5]$.

SSDL (Secondary Standard Dosimetry Laboratories) focus on mainly the calibration of devices used in the radiation therapy and radiation protection for dose measurements and put less importance on calibrations of devices used in the diagnostic radiology for dosimetric measurements. Indeed, this is due to insufficient calibration demand in the diagnostic radiology. Importance and responsibility of SSDL increase with widespread use of ionizing radiation in medical and industrial applications. Increased demand due to requirements of quality safety programs established in hospitals leads to expand calibration services, in the direction new necessities. SSDL must tend towards calibrations of mammography and CT dosimeters, as well as new metrological topics, such as various non-invasive voltage measurement devices [5].

Ionizing radiation metrology constitutes the basis to ensure reliability in occupational dose determinations of people working in the radiation field and patients participating in radiotherapy treatment or diagnostic radiology examinations, as well as in the environmental monitoring. The 
aim of reliable measurements is to establish and secure radiation protection methods to decrease the possibility of harmful biological effects caused by x-ray, beta, gamma and other ionizing radiation. However, correct measurement of radiation dose is possible by using a dosimeter calibrated at a certain radiation quality. Response of dosimeter differs depending on spectral distribution of x-ray; thus, it is of significance to determine radiation qualities in the diagnostic radiology. Radiation qualities are usually determined by x-ray tube voltage and HVL. In this regard, SSDL need to construct and secure radiation qualities and provide international comparisons and traceability to do its duty on expanding services in the diagnostic field and reducing population dose.

This study aims to calibrate dosimeters used in the diagnostic radiology of $x$-ray systems installed in SSDL, check the conformity of the quality controls of radiological systems to standards, and to generate radiation qualities that can help scientist studying in this field of the universities in accordance with the IEC 61267 standard.

\section{Materials and method}

In this study, the first target for generating diagnostic radiation qualities is to obtain RQR energy qualities. In this regard, the first priority in obtaining RQR energy qualities is to determine additional filters, as described in the IEC standard. In this study, Yxlon 160 model x-ray system, which can produce $x$-ray between $10 \mathrm{kV}$ and $160 \mathrm{kV}$, was used. Conventional diagnostic radiology uses high-voltage generators that provide a constant $\mathrm{x}$-ray tube voltage between 10 and $150 \mathrm{kV}$ to calibrate the dosimetric device in appropriate radiation qualities [4]. PTB with 1 cc volume was used in RQR energy qualities while the PTW 77334 model ion chamber and the PTW DCI 8500 model electrometer were used in dose rate measurements. IQ4 762 model dosimetry system was used as the transmission ion chamber.

One of the most important parameters in obtaining X-ray energy qualities is filtration. It is highly recommended to use high purity filter materials. In this regard, Al (aluminum) filters with $99.99 \%$ purity were used to obtain energy qualities. Spectrometric measurement the direct measurement technique of energy qualities determined by the ion chamber technique and obtained by using additional filters is achieved by using the high-purity germanium detector spectrometer.

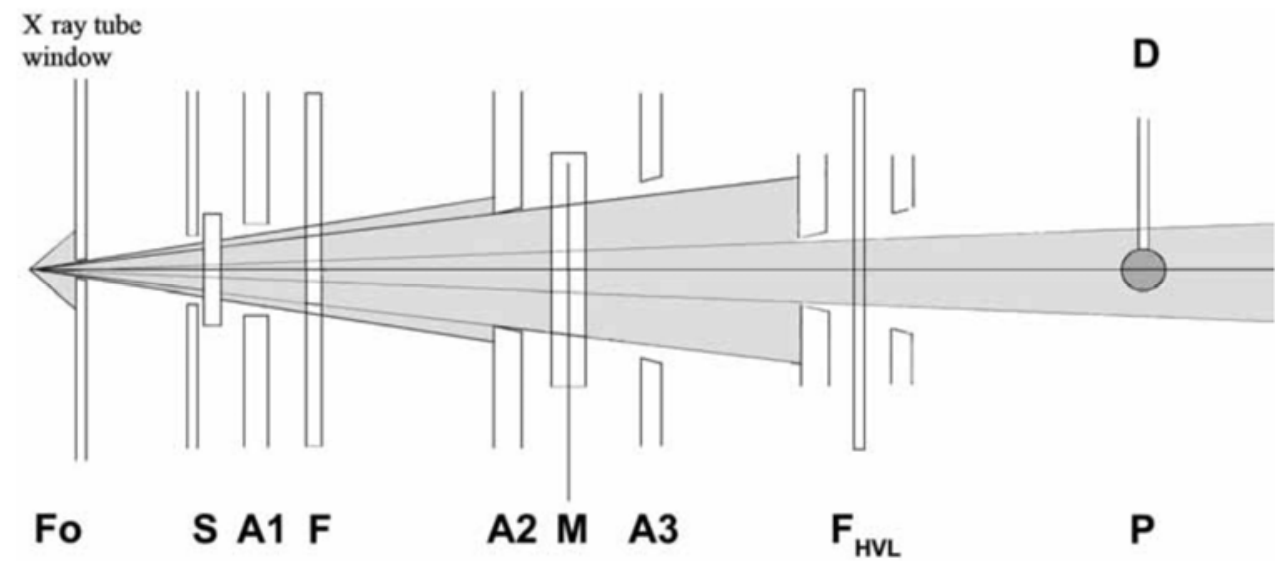

Figure 1. Schematic view of HVL measurements 
Where, $F_{o}$ is the focal point of $x$-ray; $S$ is the shutter; $F$ is the additional filter; $A_{1}, A_{2}$, and $A_{3}$ are collimators; $\mathrm{M}$ is the transmission ion chamber; $\mathrm{F}_{\mathrm{HVL}}$ is the filter setup and $\mathrm{D}$ is the ion chamber.

\section{Results}

\subsection{RQR Radiation qualities and obtainment of additional filter}

RQR radiation qualities represent the radiation beam applied to patients in general radiography, fluoroscopy and dental applications, therefore it is necessary to obtain radiation qualities in accordance with the IEC standard. Characteristic radiation qualities of the standard RQR series are shown in Table 1. The most important factor in obtaining energy qualities is additional filters because it depends on characteristics of the x-ray tube (internal filter, target material, target angle, etc.). Additional filters must primarily be identified since they may be different for each $\mathrm{x}$-ray tube. Studies were conducted when there were no additional filters in the irradiation setup shown in Figure 1. Attenuation curves of the radiation dose rate in the radiation qualities between standard dosimetry RQR2 and RQR 10 were constructed by Al filters. Figure 2 shows the attenuation curve of the dose rate and Al filter thickness of the RQR5 (70 kV \# 10mA) radiation quality.

Table 1. RQR radiation qualities and characteristics given in the IEC 61267 standard

\begin{tabular}{|c|c|c|c|}
\hline $\begin{array}{c}\text { Radiation } \\
\text { Quality IEC } \\
\mathbf{6 1 2 6 7}\end{array}$ & $\begin{array}{c}\text { X-ray } \\
\text { voltage } \\
\text { (kV) }\end{array}$ & $\begin{array}{c}\mathbf{1}^{\text {st }} \text { HVL } \\
\text { (mm Al) }\end{array}$ & $\begin{array}{c}\text { Homogeneity } \\
\text { (\%) }\end{array}$ \\
\hline RQR 2 & 40 & 1.41 & 0.81 \\
RQR 3 & 50 & 1.78 & 0.76 \\
RQR 4 & 60 & 2.19 & 0.74 \\
RQR 5 & $\mathbf{7 0}$ & $\mathbf{2 . 5 8}$ & $\mathbf{0 . 7 1}$ \\
RQR 6 & 80 & 3.01 & 0.69 \\
RQR 7 & 90 & 3.48 & 0.68 \\
RQR 8 & 100 & 3.97 & 0.68 \\
RQR 9 & 120 & 5.00 & 0.68 \\
RQR 10 & 150 & 6.57 & 0.72 \\
\hline
\end{tabular}

A rectangular template was prepared for each radiation quality by taking HVL values as reference. The rectangular template was moved over the attenuation curve to provide overlapping of opposing corners with the curve. For the energy in question, the zero point and projection on the X-axis of the rectangle were evaluated as the additional filter. Detected Al additional filters were placed in the $\mathrm{F}$ point in the setup shown in Figure 1 to confirm the validity of HVL values obtained experimentally and the HVL operation was repeated with Al filters. Amounts of additional filters obtained in nine RQR energy qualities are shown in the column 3 of the Table 2 while first and second HVL values are shown in columns 4 and 5 of the Table 2, respectively. 


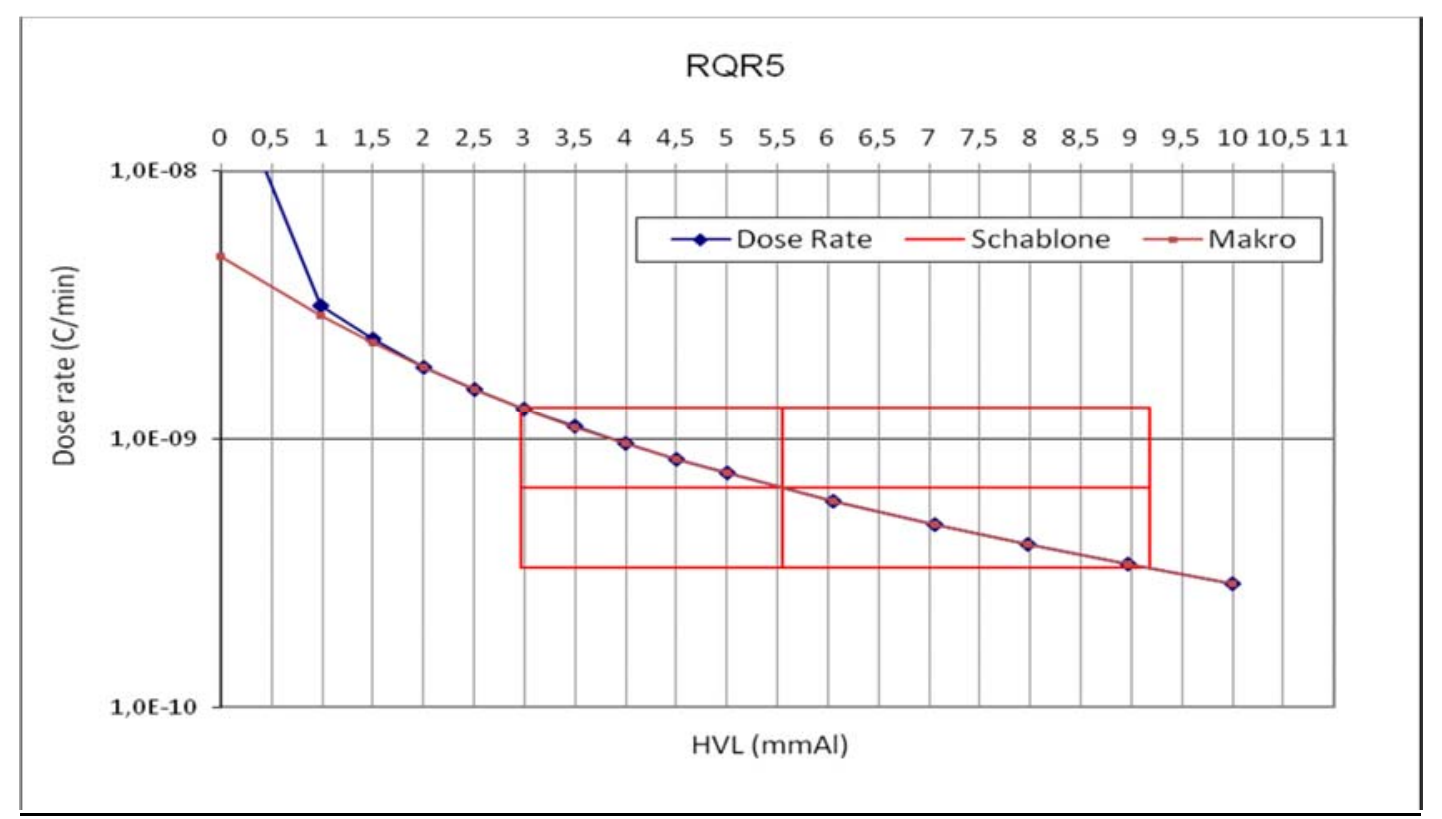

Figure 2. Additional filter determination graph for the RQR5 radiation quality

The first method to obtain radiation energy is the HVL measurement technique. This method is quite classic and troublesome. Beside this method, energy qualities can be obtained by a second method, which requires technical infrastructure and experience. A high-purity germanium detector spectrometer was used to compare energy levels obtained with the classical method and confirm the results, and spectra of each radiation quality were taken.

Table 2. RQR energy qualities and measurement results

\begin{tabular}{|c|c|c|c|c|c|c|}
\hline $\begin{array}{c}\text { Radiation } \\
\text { Quality } \\
\text { (IEC 61 267) }\end{array}$ & $\begin{array}{c}\text { X-ray } \\
\text { voltage } \\
\text { (kV) }\end{array}$ & $\begin{array}{c}\text { SSDL } \\
\text { additional } \\
\text { filter } \\
\text { (mm Al) }\end{array}$ & $\begin{array}{c}\mathbf{1}^{\text {st }} \mathbf{H V L} \\
\mathbf{( m m} \text { Al) }\end{array}$ & $\begin{array}{c}\mathbf{2}^{\text {nd }} \mathbf{H V L} \\
\mathbf{( m m} \mathbf{A l})\end{array}$ & $\begin{array}{c}\text { Attenuation } \\
\pm \mathbf{0 , 1 5} \\
\mathbf{g r a d} \text { of } \\
\mathbf{H V L}\end{array}$ & $\begin{array}{c}\text { Homogeneity } \\
\mathbf{( \% )}\end{array}$ \\
\hline RQR 2 & 40 & 2.45 & 1.41 & 1.73 & 0.502 & 0.82 \\
RQR 3 & 50 & 2.47 & 1.78 & 2.33 & 0.497 & 0.76 \\
RQR 4 & 60 & 2.76 & 2.18 & 3.00 & 0.499 & 0.73 \\
RQR 5 & 70 & 2.90 & 2.56 & 3.58 & 0.497 & 0.72 \\
RQR 6 & 80 & 3.09 & 2.98 & 4.28 & 0.506 & 0.70 \\
RQR 7 & 90 & 3.27 & 3.48 & 5.19 & 0.499 & 0.67 \\
RQR 8 & 100 & 3.36 & 3.93 & 5.89 & 0.501 & 0.67 \\
RQR 9 & 120 & 3.75 & 5.00 & 7.27 & 0.500 & 0.69 \\
RQR 10 & 150 & 4.40 & 6.54 & 9.33 & 0.498 & 0.70 \\
\hline
\end{tabular}




\subsection{RQT radiation qualities}

RQR radiation qualities form the basis of energy qualities used in diagnostic radiological applications. More clearly, to obtain RQA and RQT radiation qualities, it is sufficient to add additional filters identified for qualities specified in the IEC 61267 standard into those identified in the RQR quality and determine HVL values under these conditions. The RQA series of radiation qualities represent the radiation field behind a patient, while the RQT series represents the nonattenuated radiation beam used in CT.

At this stage of study, the setup was re-prepared by adding $0.2 \mathrm{~mm}, 0.25 \mathrm{~mm}$ and $0.3 \mathrm{~mm}$-thick $\mathrm{Cu}$ filters as specified in the IEC 61267 standard into additional filters identified in the RQR8, RQR9 and RQR10 radiation qualities of the RQR series obtained in the x-ray system. Half value thicknesses (HVLs) of RQT qualities that would be used to represent energy qualities used in CT imaging were determined by using $\mathrm{Al}$ filters. The obtained values are shown in Table 3.

Table 3. RQT Radiation qualities and measurement results

\begin{tabular}{|c|c|c|c|c|c|c|c|}
\hline $\begin{array}{l}\text { Radiation } \\
\text { qualities } \\
\text { IEC } 61267\end{array}$ & $\begin{array}{c}\text { X-ray } \\
\text { Voltage } \\
\text { kV }\end{array}$ & $\begin{array}{r}\text { SSDI } \\
\text { Additio } \\
\mathrm{mm} \mathrm{Al}\end{array}$ & $\begin{array}{l}\text { RQT } \\
\text { al filter } \\
\mathrm{mm} \mathrm{Cu}\end{array}$ & $\begin{array}{c}\text { IEC } 61267 \\
1^{\text {st }} \mathrm{HVL} \\
\mathrm{mm} \mathrm{Al}\end{array}$ & $\begin{array}{l}\text { SSDL } \\
1^{\text {st }} \mathrm{HVL} \\
\text { mm Al }\end{array}$ & $\begin{array}{c}\text { SSDL } \\
2^{\text {nd }} H V L \\
m m ~ A l\end{array}$ & Homogeneity \\
\hline RQT8 & 100 & 3.36 & 0.20 & 6.9 & 6.85 & 8.37 & 0.82 \\
\hline RQT9 & 120 & 3.75 & 0.255 & 8.4 & 8.51 & 9.99 & 0.85 \\
\hline RQT10 & 150 & 4.40 & 0.30 & 10.1 & 10.22 & 11.68 & 0.88 \\
\hline
\end{tabular}

\section{Discussions}

Radiometer and dosimetry systems are necessary in diagnostic radiology applications to accurately determine the dose measurement of patient and the radiation workers are exposed to. These systems are also safely used in measurements of both quality assurance and environmental radiation levels of ionizing radiation generating systems. Dosimetry systems that measure dose and dose rate respond highly depending on energy. Dosimetry systems that measure dose and dose rate are highly dependent on energy.

As a calibration laboratory, it is necessary to prepare the infrastructure to take measurements of radiation dosimeters and dose rate meters in accordance with standards. In this regard, the study of obtaining radiation energies used in the diagnostic radiology, ensuring reliability and supporting with a traceable secondary standard dosimeter was conducted to establish a laboratory infrastructure. The parameters mainly affecting the x-ray radiation quality are the tube voltage and first half value thickness (HVL). It is essential to determine these parameters very carefully and measure them accurately. First, additional filter determination was done according to the IEC standard, and then the results were evaluated. The IEC standard predicts HVL filtered and unfiltered air kerma dose rates to be between 0.485 and 0.515 , while in our study those values were found to be between 0.497 and 0.506 . If the ratio of radiation dose rate measurements taken with and without HVL falls within the limits specified in the standard, desired radiation qualities are said to be obtained. Radiation qualities we have achieved are within acceptable limits and very 
successful results have been achieved. The homogeneity coefficient is the quantitative that reveals the quality of the radiation obtained. In addition, in case of comparison of homogeneities, the value found in the present study shows consistency with homogeneous values suggested in the IEC standard.

It is necessary to obtain other radiation qualities, such as RQA, RQT as a result of determining RQR radiation qualities. HVL measurements were taken by adding the additional filters specified in the standard into those determined, and HVL measurements were taken and RQT radiation qualities were determined. HVL thicknesses for RQT were compared with those in the standard and it was observed that obtained values were very close to the standard values. These results are quite satisfactory.

\section{References}

[1] Bennett, B. G., "Exposures from medikal radiation worldwide. Radiat. Prot. Dosimetry" 36 (1991): 237-242.

[2] "International Commission on Radiological Protection. 1990 Recommendations of the Commission on Radiological Protection” ICRP Publication 60. Ann. ICRP 21(1990): (1-3) 42-43 (1990).

[3] "International Electrotechnical Commission. IEC 61267- medikal diagnostic x-ray equipment-Radiation conditions for use in the determination of characteristics." Geneva, Switzerland (2005).

[4] "International Organization for Standardization-ISO $4037 \mathrm{X}$ and gamma reference radiation for calibrating dosemeters and doserate meters and for determining their response as a function of photon energy." Geneva, Switzerland (1996).

[5] "IAEA Technical Reports Series No.: 457Dosimetry in diagnostic radiology." An international code of practice 2007. 